\title{
Spatial analysis increases efficiency of progeny testing of Chinese fir
}

\author{
Liming Bian ${ }^{1} \cdot$ Renhua Zheng ${ }^{2} \cdot$ Shunde $\mathrm{Su}^{2} \cdot$ Huazhong $\mathrm{Lin}^{3} \cdot \mathrm{Hui} \mathrm{Xiao}^{2} \cdot$ \\ Harry Xiaming $\mathrm{Wu}^{4} \cdot$ Jisen $\mathrm{Shi}^{5}$
}

Received: 19 March 2016/Accepted: 30 March 2016/Published online: 19 November 2016

(C) The Author(s) 2016. This article is published with open access at Springerlink.com

\begin{abstract}
We used spatial, global trend and post-blocking analysis to examine the effectiveness of a progeny trial in a tree breeding program for Chinese fir (Cunninghamia lanceolata (Lamb.) Hook) on a hilly site with an environmental gradient from hill top to bottom. Diameter at breast height (DBH) and tree height data had significant spatial auto-correlations among rows and columns. Adding a firstorder separable autoregressive term more effectively modelled the spatial variation than did the incomplete block (IB) model used for the experimental design. The spatial model
\end{abstract}

Project funding: This study was funded by the Natural Science Foundation of the Jiangsu Higher Education Institutions of China (Grant No. 15KJA220002), Priority Academic Program Development of Jiangsu Higher Education Institutions (PAPD), and Fujian Province Science and Technology Research funding on the fourth Tree Breeding Cycle Program of Chinese fir (Grant No. Min Lin 2016-1).

The online version is available at http://www.springerlink.com

Corresponding editor: Yu Lei

Harry Xiaming Wu

Harry.wu@slu.se

1 South China Forestry Multidisciplinary Collaborative Innovation Center, College of Forestry, Nanjing Forestry University, 159 Longpan Road, Nanjing 210037, People's Republic of China

2 Fujian Academy of Forestry, 35 Shangchiqiao, Fuzhou 350012, People's Republic of China

3 Jiangle Forest Farm, Jiangle 353300, People's Republic of China

4 Department of Forest Genetics and Plant Physiology, Umeå Plant Science Centre, Swedish University of Agricultural Sciences, 90183 Umeå, Sweden

5 College of Forestry, Nanjing Forestry University, 159 Longpan Road, Nanjing 210037, People's Republic of China also accounted for effects of experimental design factors and greatly reduced residual variances. The spatial analysis relative to the IB analysis improved estimation of genetic parameters with the residual variance reduced 13 and 19\% for DBH and tree height, respectively; heritability increased 35 and $51 \%$ for DBH and tree height, respectively; and genetic gain improved $3-5 \%$. Fitting global trend and postblocking did not improve the analyses under IB model. The use of a spatial model or combined with a design model is recommended for forest genetic trials, particularly with global trend and local spatial variation of hilly sites.

Keywords Chinese fir - Genetic variance - Heritabilities · Progeny testing $\cdot$ Spatial analysis

\section{Introduction}

The accurate prediction of breeding values and estimation of genetic parameters in tree breeding program rely on well-designed progeny trials. Progeny trials for forest tree species are usually characterized by large field size, heterogeneous global and microenvironmental variation and long-term observations. Various experimental designs were proposed to separate within-site micro-environmental variation from the treatment variation as much as possible (Cochran and Cox 1957). Randomized complete block (RCB), set-in-block, and noncontiguous plots were initially used in forestry field trials. Later, incomplete block designs including row-column design were introduced to reduce the size of replicated block (Libby and Cockerham 1980; Williams et al. 2002; Patterson and Thompson 1971). To further separate microenvironmental variation from treatment variation, single-tree plots were recommended over the usual multiple-tree row plots (Loo-Dinkins 1992). 
To deal with spatial correlation between adjacent plots, nearest neighbor methods were first established to take such variation into account (Papadakis 1937; Bartlett 1938). In crops and forest trees, spatial or neighbor adjustment were proposed more than a quarter of century ago (Gleeson and Cullis 1987; Martin 1990; Magnussen 1990, 1993; Wilkinson et al. 1983). But a spatial model was only systematically applied in the last decades thanks to the development of software such as ASReml to deal with complex linear mixed models (Gilmour et al. 2009; Smith et al. 2005; Gilmour and Cullis 1995). Spatial analyses could improve the estimation of genetic effects by modeling the spatial distribution of error effects and separating spatially correlated global and microsite variation from independent random environmental variation in forestry progeny trials. Previous studies have demonstrated that site variation in many forest genetic trials is generally spatially continuous, and residuals from close locations are more related than distant locations. By introducing spatial models into the analysis of forest genetic tests for tree height, Magnussen (1990) reported a reduction of 5-20\% in the standard error of the difference between full-sib families and an increase in the number of significant differences among treatments, relative to the RCB design (Magnussen 1990). Kusnadar and Galwey (2000) showed that a spatial model gave a significantly better fit than an incomplete block model for tree growth traits in maritime pine (Pinus pinaster Ait.). On the basis of 12 forest tree progeny trials, Costa e Silva et al. (2001) concluded that a spatial model increased REML (restricted maximum likelihood) log-likelihood and the prediction accuracy of genetic values. Dutkowski et al. (2002, 2006) presented one of most convincing evidence using 55 trials in 6 species that spatial analysis methods improved predicted genetic responses by more than $10 \%$ for 20 variables and recommended that spatial analysis should be routinely applied in forest genetic trial analyses where the spatial arrangement of trees can be determined. Ye and Jayawickrama (2008) reported that more than $97 \%$ of the data sets in 275 trials had significantly improved by spatial analysis, and $14-34 \%$ of the residual variances could be removed by fitting a spatial correlation model. This improved the accuracy of breeding value prediction up to $20 \%$.

Chinese fir (Cunninghamia lanceolata (Lamb.) Hook) is the predominant commercial plantation species in China, accounting for about $25 \%$ timber production and $6.7 \%$ of the total area of forest resources. Breeding for growth traits in the first two generations has increased average growth rate by almost 30\% (Shi 1994). In a quantitative genetics survey, several unusual and interesting unique genetic properties of Chinese fir's breeding population such as low heritability of wood density and low negative genetic correlation between growth and wood density, a large difference between narrow-sense and broad-sense heritability, and regional-related $\mathrm{G}$ by $\mathrm{E}$ interactions were found (Bian et al. 2014). Several relatively large progeny trials with a family of sufficient size for genetic analyses revealed low heritability for DBH (e.g., $h^{2}=0.09$ in a trial of 112 families (Fang and Shi 1998); $h^{2}=0.05$ in another trial of 228 families (Yu et al. 2001)). The typical progeny test site for Chinese fir in southern China is planted on a hilly slope with a usual global environment gradient from the hill top to valley. Planting blocks are usually arranged on the similar contour along the hill, creating a potential spatial correlation among neighboring trees on the similar contour of the same block. Therefore, proper experimental design and an adequate analytical model to reduce correlated environmental variation are probably more important for progeny testing than planting on a flatter site. In this study, we will explore the utility of spatial analyses in reducing the environmental variation and increase of heritability estimates (e.g., increasing the efficiency of progeny analyses) in Chinese fir progeny trials in an approach similar to that used by Costa e Silva et al. (2001), Dutkowski et al. (2002, 2006), and Ye and Jayawickrama (2008). We also used post-blocking and fitted global trend to compare with spatial analyses and whether post-blocking and fitted global trend are similarly efficient in reducing environmental variance as the spatial model. The DBH from a single site in northern Fujian of China was used to evaluate the efficiency of a spatial two-dimensional autocorrelated model, post-blocking, and fitting global trend over incomplete block (IB) analysis by assessing the reduction in residual variance, changes in heritability, and genetic gain.

\section{Materials and methods}

\section{Materials}

The study was based on a progeny trial at Weimin Forest Farm (latitude $27^{\circ} 05^{\prime} \mathrm{N}$; longitude $117^{\circ} 40^{\prime} \mathrm{E}$ ), Fujian Province planted in February 2003 with 80 open-pollinated families and one control, using an incomplete block experimental design with 10 replications. There were nine incomplete blocks per replication with nine plots per block, and four trees in a row-plot along the contour (e.g., the row is perpendicular to the slope). Tree spacing was $2 \mathrm{~m} \times 2 \mathrm{~m}$ with a buffer zone established around the trial. The half-sib families were derived from Nanjing Forestry UniversityFujian Province Chinese Fir Cooperation second-generation breeding population at Guanzhuang Forest Farm (latitude $26^{\circ} 32^{\prime} \mathrm{N}$; longitude $117^{\circ} 45^{\prime} \mathrm{E}$ ). The planting site was on a relatively steep hill of $23^{\circ}$ slope, and the previous crop was a Chinese fir plantation. The site was relative fertile with a 
layer of $20 \mathrm{~cm}$ humus above the base red soil about $90 \mathrm{~cm}$ deep before planting. Tree height and diameter at $1.3 \mathrm{~m}$ above ground $(\mathrm{DBH})$ were measured during the growing season of 2005-2011, equivalent to a stand age of 3-9 years after planting. For facilitating spatial analysis, every tree was uniquely identified by a row and column position in a total of 56 rows and 171 columns (Fig. 1a).

\section{Statistical model and analysis}

The following linear mixed model was used in the genetic analysis of the trial:

$y=X b+Z u+e$,

where, $y$ is the vector of individual tree observations; $b$ is the vector of fixed effects with the overall mean as the first element; $u$ is the vector of random effects of individual trees including replications, incomplete blocks, plots and additive genetic merits; $e$ is the vector of random residual deviations of individual trees; $X$ and $Z$ are known design matrices for fixed and random effects, respectively. Separate model terms in $u$ were assumed to be uncorrelated. Fixed and random effect solutions are obtained by solving the mixed model equations:

$$
\left[\begin{array}{ccc}
X^{\prime} & R^{-1} X & X^{\prime} R^{-1} Z \\
Z^{\prime} & R^{-1} X & Z^{\prime} R^{-1} Z+G^{-1}
\end{array}\right]\left[\begin{array}{l}
\hat{b} \\
\hat{u}
\end{array}\right]=\left[\begin{array}{cc}
X^{\prime} & R^{-1} y \\
Z^{\prime} & R^{-1} y
\end{array}\right]
$$

where, $R$ is the variance-covariance matrix of residuals and $\mathbf{G}$ is the direct sum of the variance-covariance matrices of each of the random effects. Residuals are assumed to be independent, and $\mathbf{R}$ is denoted as $\sigma_{\mathrm{e}}^{2} \mathbf{I}$, but spatial analysis allows $\mathbf{R}$ to have a different structure based on a decomposition of $\mathbf{e}$ into spatially dependent $(\boldsymbol{\xi})$ and spatial independent $(\boldsymbol{\eta})$ residuals (nugget effect) to model a correlation structure in correlated residual variance. In our analyses, $\xi$ was modelled as a first-order separable

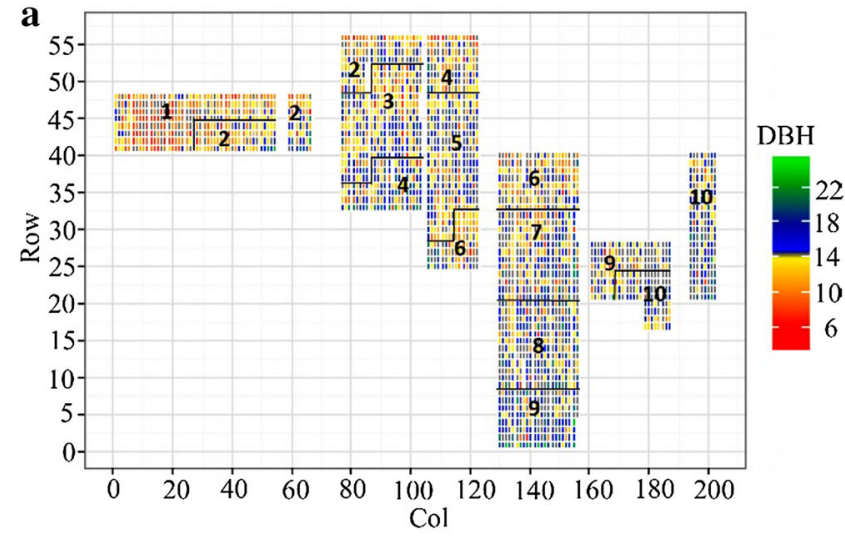

Fig. 1 a DBH distribution at age 9 along the row and columns (Numbers in the figure indicate replicates and the rows are perpendicular to the slope). b Independent residual distribution of DBH at autoregressive process $(\mathbf{A R} 1 \otimes \mathbf{A R} 1)$ with the variancecovariance matrix structured as:

$$
\begin{aligned}
& \operatorname{Var}(\boldsymbol{\xi})=\sigma_{\xi}^{2}\left[\mathbf{A R} 1\left(\rho_{\mathrm{col}}\right) \otimes \mathbf{A R} 1\left(\rho_{\text {row }}\right)\right] \\
& \mathbf{A R} 1\left(\rho_{\text {col }}\right)=\left[\begin{array}{ccccc}
1 & \rho_{c} & \rho_{c}^{2} & \cdots & \rho_{c}^{n_{c}^{-1}} \\
\rho_{c} & 1 & \rho_{c} & \cdots & \rho_{c}^{n_{c}^{-2}} \\
\rho_{c}^{2} & \rho_{c} & 1 & \cdots & \rho_{c}^{n_{c}^{-3}} \\
\vdots & \vdots & \vdots & \ddots & \vdots \\
\rho_{c}^{n_{c}^{-1}} & \rho_{c}^{n_{c}^{-2}} & \rho_{c}^{n_{c}^{-3}} & \cdots & 1
\end{array}\right] \\
& \mathbf{A R} 1\left(\rho_{\text {row }}\right)=\left[\begin{array}{ccccc}
1 & \rho_{r} & \rho_{r}^{2} & \cdots & \rho_{r}^{n_{r}^{-1}} \\
\rho_{r} & 1 & \rho_{r} & \cdots & \rho_{r}^{n_{r}^{2}} \\
\rho_{r}^{2} & \rho_{r} & 1 & \cdots & \rho_{r}^{n_{r}^{-3}} \\
\vdots & \vdots & \vdots & \ddots & \vdots \\
\rho_{r}^{n_{r}^{-1}} & \rho_{r}^{n_{r}^{-2}} & \rho_{r}^{n_{r}^{-3}} & \cdots & 1
\end{array}\right]
\end{aligned}
$$

where, $\sigma_{\xi}^{2}$ is the spatial variance of the trend process and Eqs. 4 and 5 are the first order autoregressive correlation matrices with autocorrelation parameters $\rho_{\text {col }}$ and $\rho_{\text {row }}$ for $n_{\mathrm{c}}$ columns and $n_{\mathrm{r}}$ rows, respectively, and $\otimes$ is the Kronecker product. The elements of vector $\boldsymbol{\eta}$ are assumed to be pairwise independent, so the residual variance-covariance matrix of the spatial model is defined as:

$\operatorname{Var}(\mathbf{e})=\mathbf{R}=\sigma_{\xi}^{2}\left[\mathbf{A R} 1\left(\rho_{\text {col }}\right) \otimes \mathbf{A R} 1\left(\rho_{\text {row }}\right)\right]+\sigma_{\eta}^{2} \mathbf{I}_{n_{c} * n_{r}}$,

where, $\sigma_{\eta}^{2}$ is the variance of the independent residuals and $\mathbf{I}$ is an identity matrix. The residuals in $\boldsymbol{\eta}$ reflect random environmental effects after accounting for correlated spatial variation.

Global model and post-blocking model were also fitted along with the design model to examine the efficiency at

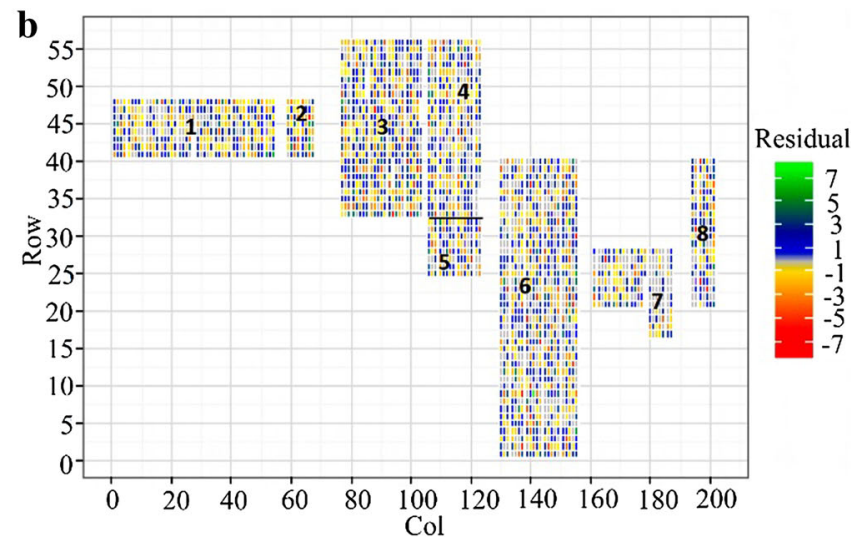

age 9 after fitting spatial model and eight post-blocks are shown on the figure. Note the color of grey means missing values 
removing the environmental variation from the residual variance. We first examined whether the designed factor, such as replication, had significant effect to reduce environmental variation in the trial. Then we used post-blocking to examine its effectiveness in reducing environmental variation. Finally, we fitted the spatial model to further study the effectiveness of the spatial model in reducing environmental variation. Therefore, five models with various combinations of random effects and definitions of $\mathbf{R}$ and $\mathbf{G}$ were fitted for the trial: (1) a design base model (D) which fitted replication, incomplete block, and plot as random effects; (2) design base model plus post-blocking (DP) where a new random incomplete block term was fitted in addition to the base model (Eight post-blocks were selected based on the DBH pattern and the location of each natural block as illustrated in Fig. 1b); (3) spatial model with first-order autoregressive for both row and column (S); (4) design base model plus global trend (DG) in which a second degree of polynomial was fitted along the row and column; (5) spatial model plus global trend (GS).

For testing the significance of the random term, the ratio of the variance component to their corresponding standard error was used. If the ratio was larger than 2 , then we regarded the random term as significant. If the ratio was less than 1 , then we regarded the random term not significant. For ratios between 1 and 2, a likelihood ratio test (LRT) was applied assuming that log-likelihood ratio LLRT [ $=-2 \times$ (difference of log-likelihood excluding and including the random term)] follows a $\chi_{0.5}^{2}$ distribution with sufficient large size of sample (Gilmour et al. 2009). Spatial models were fitted from age 3-9 (after planting) for both height and DBH to examine a possible time trend of autocorrelation of spatial variation.

Assuming spatial variance could be separated as part of a correlated environmental variance, independent residual variances were used to estimate individual tree narrow sense heritability $\left(h^{2}\right)$ for tree height and DBH:

$\hat{h}^{2}=\frac{\hat{\sigma}_{A}^{2}}{\hat{\sigma}_{A}^{2}+\hat{\sigma}_{\mathrm{e}}^{2}}$

where, $\sigma_{A}^{2}$ is additive genetic variance. Genetic gain was estimated using Eq. 8:

$\Delta G=\frac{i \times h^{2} \times \sigma_{p}}{M} \times 100$

where, $i$ is the selection intensity, $h^{2}$ is the narrow-sense heritability, $\sigma_{\mathrm{p}}$ is the phenotypic standard deviation, and $M$ is the mean value of trait.

Variance parameters were estimated by the restricted maximum likelihood (REML) method and an approximate standard error of statistics was obtained by Taylor expansion, implemented in the ASReml software package (Gilmour et al. 2009).
The Akaike information criterion (AIC) was calculated for model selection as:

$\mathrm{AIC}=-2 \ln L+2 k$

where, $\ln L$ is the maximized value of the likelihood function for the mode, $k$ is the number of estimated variance parameters in the model. And the preferred model is the one with the minimum AIC value.

\section{Results}

The mortality in the trial at the final measurement of age 9 after planting was around $25.2 \%$. The average height and DBH reached $8.4 \mathrm{~m}$ and $13.5 \mathrm{~cm}$ with corresponding standard deviation of $1.4 \mathrm{~m}$ and $3.4 \mathrm{~cm}$, respectively (Table 1). The histogram suggested that the data are approximately normally distributed.

Five models were fitted for both DBH and tree height and all nonsignificant effects removed from the models except for post-block variances. Table 2 presents results for DBH for the designed factors (e.g., replicates, incomplete blocks, and plots) in five models. All design factors were significant $(p<0.05)$ for DBH from age 3-9 in the model using experiment design (Design). However, postblocks were not significant at all ages for DBH. When an autoregressive spatial correlation and independent error were fitted together with the design factors, the other design factors were insignificant at most ages except at age 4, 5, and 7 for plots and at age 3, 4, and 5 for incomplete blocks. These results may indicate that the replicate, incomplete block and plot effect were absorbed by the spatial variances at most ages, particularly at late ages.

Distribution of individual tree growth at age 9 is shown in Fig. 1a. The spatial pattern for DBH had an apparent global trend of increasing tree size from the top to the bottom of the hill. The residual was more random after fitting the spatial model for the same data at age 9 (Fig. 1b). Figure 2 presents variograms for DBH and tree height at age 9 and shows typical highly correlated spatial variation for both row and column, with trees that are closer together being more similar than those that are farther apart.

Both spatial model and spatial plus global model increased additive genetic variances relative to the experimental design model, while post-blocking and adding global trend under the design model did not increase the additive genetic variance (Fig. 3a). Also the increase in the additive genetic variance was not obvious at age 3 , but it increased as trees grew from age 4-9. Similarly, independent residual variances were reduced after fitting the spatial and the spatial plus global model while fitting of post-block and global model did not reduce the independent residual 
Table 1 The mortality, overall mean value of height and $\mathrm{DBH}$ at different stand age with approximate standard deviation in parentheses

\begin{tabular}{lllc}
\hline Stand age (year) & Mortality $(\%)$ & Mean value of height $(\mathrm{m})$ & Mean value of DBH $(\mathrm{cm})$ \\
\hline 3 & 23.1 & $2.2(0.5)$ & $2.1(1.1)$ \\
4 & 24.2 & $3.9(0.8)$ & $5.7(1.9)$ \\
5 & 24.3 & $4.9(0.9)$ & $6.8(2.1)$ \\
6 & 24.4 & $5.9(1.1)$ & $9.5(2.7)$ \\
7 & $7.0(1.2)$ & $10.9(2.9)$ \\
8 & 24.5 & $8.0(1.4)$ & $12.3(3.2)$ \\
9 & 25 & $8.4(1.4)$ & $13.5(3.4)$ \\
\hline
\end{tabular}

Table 2 Estimated variances of design factors using five models ( $D$ Design, $D P$ Design plus Post-blocking, $S$ Spatial, $D G$ Design plus Global, GS Spatial plus Global) for DBH of Chinese fir trial from age 3-9

\begin{tabular}{|c|c|c|c|c|c|c|c|c|c|}
\hline $\begin{array}{l}\text { Age and } \\
\text { model }^{\mathrm{a}}\end{array}$ & Post-block & Replicate & Plot & $\begin{array}{l}\text { Incomplete } \\
\text { block }\end{array}$ & Age and model & Post-block & Replicate & Plot & $\begin{array}{l}\text { Incomplete } \\
\text { block }\end{array}$ \\
\hline $3 \mathrm{D}$ & & $0.17(0.09)$ & $0.05(0.02)$ & $0.22(0.04)$ & $7 \mathrm{D}$ & & $1.75(0.90)$ & $0.40(0.14)$ & $1.13(0.22)$ \\
\hline $3 \mathrm{DP}$ & $0.11(0.09)^{\mathrm{ns}}$ & $0.17(0.10)$ & $0.05(0.02)$ & $0.17(0.04)$ & $7 \mathrm{DP}$ & $0.45(0.40)^{\mathrm{ns}}$ & $1.66(0.93)$ & $0.40(0.14)$ & $0.95(0.20)$ \\
\hline $3 \mathrm{~S}$ & & & & $0.03(0.02)$ & $7 \mathrm{~S}$ & & & $0.24(0.13)$ & \\
\hline $3 \mathrm{G}$ & & $0.13(0.08)$ & $0.05(0.02)$ & $0.17(0.03)$ & $7 \mathrm{G}$ & & $1.30(0.78)$ & $0.40(0.14)$ & $0.77(0.17)$ \\
\hline $3 \mathrm{GS}$ & & & & $0.02(0.02)$ & 7GS & & & $0.23(0.13)$ & \\
\hline $4 \mathrm{D}$ & & $0.68(0.36)$ & $0.19(0.06)$ & $0.52(0.10)$ & $8 \mathrm{D}$ & & $1.88(0.96)$ & $0.40(0.16)$ & $1.13(0.23)$ \\
\hline $4 \mathrm{DP}$ & $0.21(0.18)^{\mathrm{ns}}$ & $0.61(0.35)$ & $0.19(0.06)$ & $0.44(0.09)$ & $8 \mathrm{DP}$ & $0.46(0.41)^{\mathrm{ns}}$ & $1.77(0.99)$ & $0.40(0.16)$ & $0.96(0.21)$ \\
\hline $4 \mathrm{~S}$ & & & $0.13(0.06)$ & $0.10(0.05)$ & $8 \mathrm{~S}$ & & & & \\
\hline $4 \mathrm{G}$ & & $0.42(0.25)$ & $0.19(0.06)$ & $0.40(0.08)$ & $8 \mathrm{G}$ & & $1.53(0.94)$ & $0.41(0.16)$ & $0.75(0.18)$ \\
\hline 4GS & & & $0.13(0.06)$ & $0.08(0.05)$ & $8 \mathrm{GS}$ & & & & \\
\hline $5 \mathrm{D}$ & & $0.90(0.47)$ & $0.23(0.07)$ & $0.72(0.14)$ & 9D & & $1.95(0.99)$ & $0.38(0.19)$ & $1.08(0.23)$ \\
\hline $5 \mathrm{DP}$ & $0.46(0.33)^{\mathrm{ns}}$ & $0.67(0.40)$ & $0.23(0.07)$ & $0.54(0.11)$ & 9DP & $0.46(0.41)^{\mathrm{ns}}$ & $1.75(0.98)$ & $0.38(0.19)$ & $0.91(0.21)$ \\
\hline $5 \mathrm{~S}$ & & & $0.16(0.06)$ & $0.13(0.06)$ & $9 \mathrm{~S}$ & & & & \\
\hline $5 \mathrm{G}$ & & $0.55(0.33)$ & $0.23(0.07)$ & $0.53(0.11)$ & $9 \mathrm{G}$ & & $1.35(0.84)$ & $0.39(0.19)$ & $0.70(0.18)$ \\
\hline $5 \mathrm{GS}$ & & & $0.15(0.06)$ & $0.10(0.06)$ & 9GS & & & & \\
\hline $6 \mathrm{D}$ & & $1.52(0.78)$ & $0.32(0.11)$ & $1.07(0.20)$ & & & & & \\
\hline $6 \mathrm{DP}$ & $0.35(0.34)^{\mathrm{ns}}$ & $1.40(0.79)$ & $0.32(0.11)$ & $0.94(0.19)$ & & & & & \\
\hline \multicolumn{10}{|l|}{$6 \mathrm{~S}$} \\
\hline $6 \mathrm{G}$ & & $1.12(0.68)$ & $0.33(0.11)$ & $0.78(0.16)$ & & & & & \\
\hline $6 \mathrm{GS}$ & & & & & & & & & \\
\hline
\end{tabular}

${ }^{a}$ All non-significant effects were removed in the fitted model except for post-blocking effect, and the approximate standard errors for the estimated parameters are given in parenthesis

$n s$ non-significant $(p>0.05)$

variances (Fig. 3b). As a consequence, heritability for DBH increased between 23 and $31 \%$ for ages 3-5, and between 31 and $64 \%$ for ages $6-9$ using the spatial model relative to experimental design model (Fig. 3c). Models using post-blocking and global trend were found to have no effect on heritability relative to the design models.

The log-likelihoods increased after fitting the spatial model between 50 and 80 and were highly significant $(p<0.001)$ relative to the base design model (Fig. 3d). Similarly, the AIC decreased between 98 and 157 relative to the base design model with significant effects for all ages (Fig. 3e).
The age trends for autocorrelation are shown in Fig. 4a. Columns had higher spatial correlation than rows. Fitting of the spatial plus global model slightly decreased autocorrelation with slightly more decrease in rows than in columns. In addition, the independent residual variance and spatial and additive variances all increased with age, with the residual and the spatial variance rising the quickest. The spatial variance in the global plus spatial model increased slower than the corresponding one in the spatial model as trees aged (Fig. 4b).

Overall, age trends for the five models of tree height were similar to DBH. On average, heritability increased 
a means missing values.

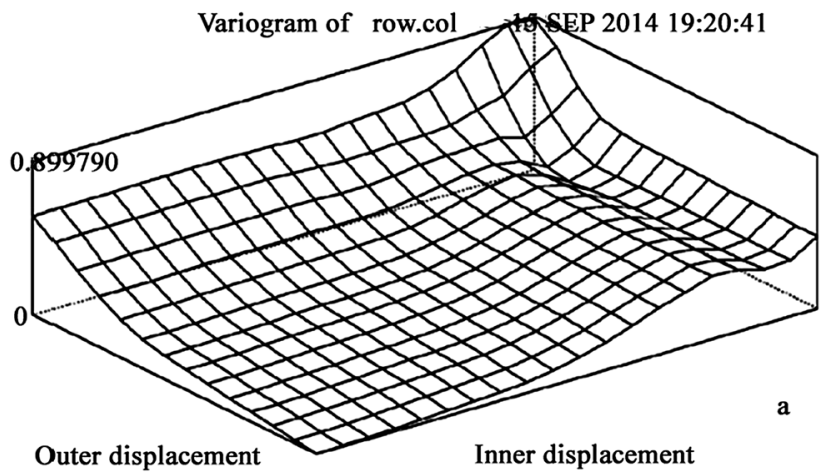

b

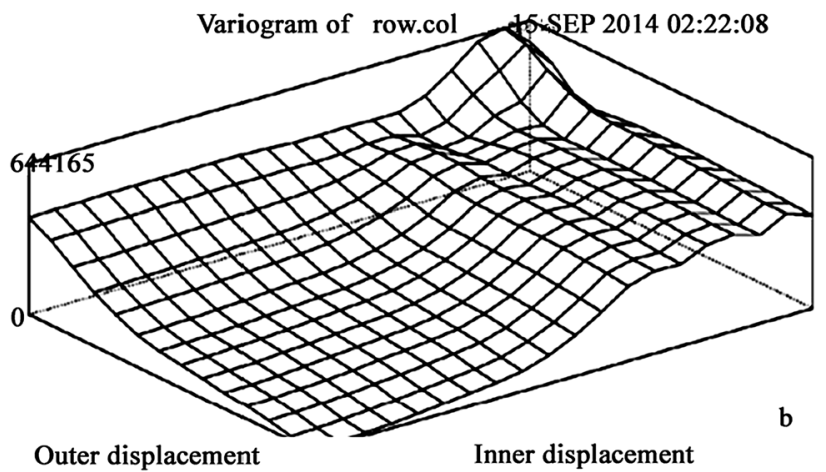

Fig. 2 Variogram of residual for DBH and tree height (Height) at 9 year-old after planting
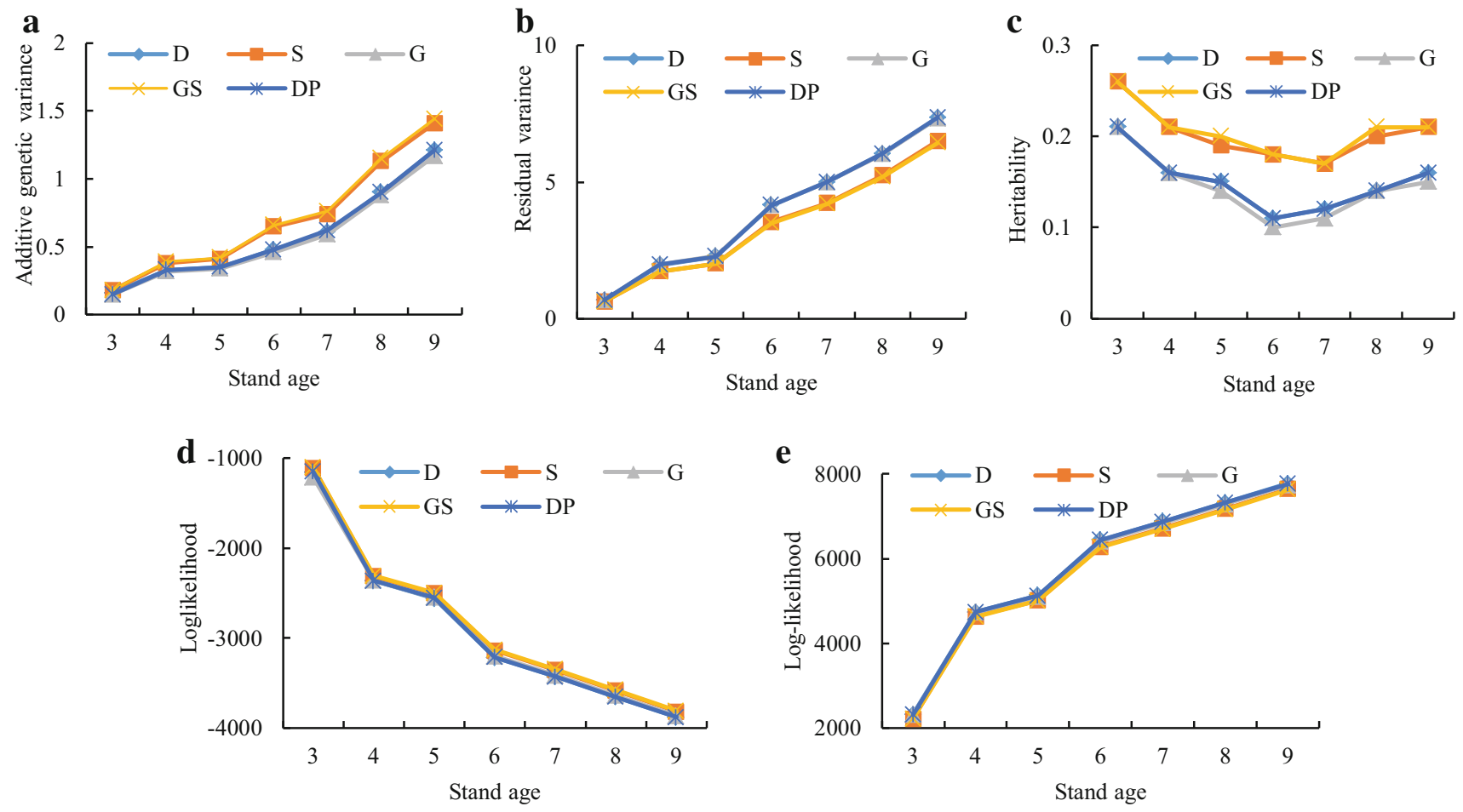

Fig. 3 Age trends of a Additive genetic variance, b Residual variance, c Heritability, d Log-likelihood, e Akaike information criterion (AIC) based on five models ( $D$ Design, DP Design plus Post-

blocking, $S$ Spatial, $D G$ Design plus Global, $G S$ Spatial plus Global) for DBH of Chinese fir trial

from 0.15 to 0.20 for $\mathrm{DBH}$, and from 0.19 to 0.28 for tree height with a 35 and $52 \%$ increase, respectively.

The relative genetic gains from parental and mass selection also increased using the spatial model relative to the design model. For example, the gain for DBH increased about 3.2 and $5.2 \%$ from using the spatial analysis relative to the experimental design model when selection intensity was $10 \%$ for parents and $0.5 \%$ for mass selection.

\section{Discussion and conclusion}

The main objective of a progeny trial in a tree breeding program is to estimate genetic parameters and breeding values for selection. For reducing environmental variance due to environmental heterogeneity in the progeny trial, various experimental designs have been suggested. The simplest experimental design is a completely randomized design, which was adopted by northern Swedish tree 

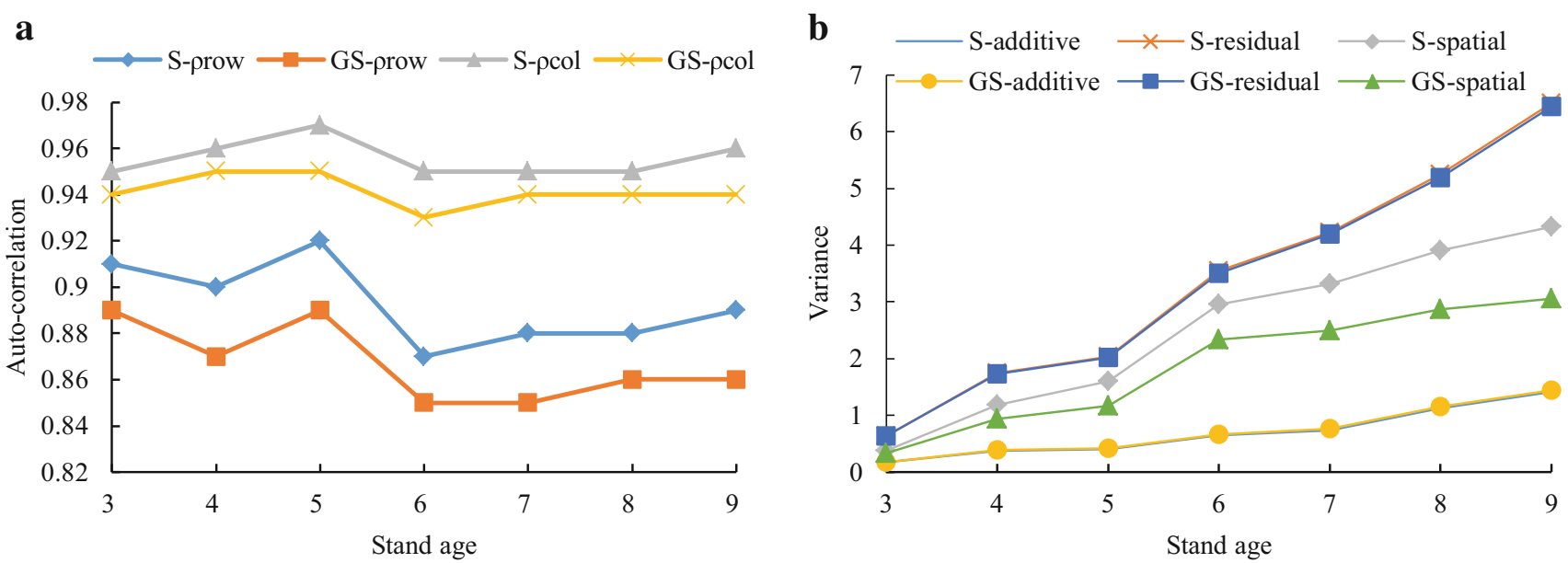

Fig. 4 a Age trend of row ( $\rho r o w)$ and column ( $\rho$ col) auto-correlation under two models ( $S$ Spatial, GS Spatial plus Global) and b Spatial, additive and residual variance under Spatial model (S) and spatial variance under Spatial plus Global model (GS) for DBH of Chinese fir trial

breeders. However, these tree breeders successfully used post-blocking to reduce the environmental variation associated with large blocks (Dutkowski et al. 2002; Ericsson 1997). The most common experimental design is a randomized complete block design (Loo-Dinkins 1992; White et al. 2007). For further reducing environmental heterogeneity, an incomplete block design was introduced, particularly with a row and column design (Loo-Dinkins 1992). Incomplete block designs were adopted for controlling within-site variation in most Chinese-fir progeny trials planted in the 1990s and 2000s. Usually 50-120 families of 2-4 trees per row-plot were deployed in Fujian Chinese fir breeding testing. The incomplete block design was generally effective in Fujian's hilly Chinese fir sites. These sites typically have environmental gradients from hill top to bottom (Bian et al. 2014). To reduce this global trend of environmental variation, blocks were usually employed along the contour of the site to keep the microenvironment within the same block as homogeneous as possible. Such sites may be well suited for spatial analyses to reduce correlated environmental variation along rows and columns.

Our analysis revealed that both adding a global trend and imposing post-blocking was not effective under the experimental design model using an incomplete block on this hilly Chinese fir site. However, spatial analyses increased efficiency and not only accounted for the effects of design factors (replicates and incomplete blocks), but also reduced residual variance by separate spatial variation from the independent residual variation. Independent residual variation was markedly reduced by the spatial autocorrelation analysis, which may indicate that part of the environmental variation was autocorrelated and that spatial analyses can separate it from the independent environmental variation. Furthermore, spatial analyses also accounted for the large global field variation from the hill top to the bottom. Replication is traditionally used to account for this global trend in the experimental design in hilly sites.

Spatial autocorrelation was significant at an early age, 3 years, in the Chinese-fir experiment, but all parameters (e.g., additive and residual variances, log-likelihood and AIC) indicated that spatial analyses were more effective for DBH from age 4 onward. There were higher autocorrelations among columns than rows, which is not surprising since adjacent trees among the columns were planted on the same contour, while adjacent trees among rows were planted along an altitudinal gradient. Therefore, the autocorrelation among rows may account for the global trend from hill top to bottom, while autocorrelation among columns may account for local spatial variation, e.g., the similarity in the environments on the same contour of the terraces.

All 14 spatial models for DBH and tree height improved heritability in this study, which was similar to observations in Douglas-fir (Pseudotsuga menziesii) in which $97 \%$ of the data sets showed significant model improvement using spatial analysis. An improvement in model fit using spatial analysis was also observed in $90 \%$ of the cases for other tree species such as Picea abies, Picea mariana, Picea sitchensis, Pinus pinaster, Pinus radiata, and Eucalyptus globulus (Dutkowski et al. 2006; Ye and Jayawickrama 2008).

In our experiment using an incomplete block design, residual variance was the dominant term. In the spatial model, the independent residual was reduced by $13 \%$ for DBH and $19 \%$ for tree height on average. These were similar to the Doulas-fir study for which, on average, spatial analyses removed $14-34 \%$ of the correlated variance due to spatial correlation (Ye and Jayawickrama 
2008). Narrow-sense heritabilities for individual tree increased 35 and $51 \%$ on average by removing spatial variance from the residual variance, which was higher than the results for DBH summarized by Dutkowski et al. (2006). This result is not surprising considering that both high autocorrelations in rows and columns were observed in this hilly site, perhaps indicating that the spatial analyses are more useful or powerful for hilly sites such as the Chinese-fir sites. However, we only analyzed one site. If more sites are found with such high two-dimensional autocorrelations, then spatial analyses should routinely be applied to similar forest progeny testing sites in countries where most forest trials are on hilly sites, such as in southern China and other southeastern Asian countries.

A moderate genetic gain of about $3-5 \%$ for selection was determined in this study using spatial analyses, similar to the finding that extra genetic gain usually did not exceed 6\% (Dutkowski et al. 2002). However higher gain was observed by Costa e Silva et al. (2001) in several progeny trials including radiate and maritime pines and Sitka spruce. In conclusion, fitting with global trend and postblocking was not effective with the current incomplete block design used in Chinese-fir progeny trial, while spatial analyses reduced residual variance, increased heritability and genetic gain.

Acknowledgements Thanks to Weimin National Forest Farm for trial maintenance over the years. Special thanks are extended to Dr. Gregory W. Dutkowski for helpful comments. Drs. Li Li and Miloš Ivković are also thanked for useful insights.

Open Access This article is distributed under the terms of the Creative Commons Attribution 4.0 International License (http://creative commons.org/licenses/by/4.0/), which permits unrestricted use, distribution, and reproduction in any medium, provided you give appropriate credit to the original author(s) and the source, provide a link to the Creative Commons license, and indicate if changes were made.

\section{References}

Bartlett MS (1938) The approximate recovery of information from replicated field experiments with large blocks. J Agric Sci 28:418-427

Bian L, Shi J, Zheng R, Chen J, Wu HX (2014) Genetic parameters and genotype-environment interactions of Chinese fir (Cunninghamia lanceolata) in Fujian province. Can J For Res 44:582-592

Cochran WG, Cox GM (1957) Experimental designs, 2nd edn. Wiley, New York

Costa e Silva J, Dutkowski GW, Gilmour AR (2001) Analysis of early tree height in forest genetic trials is enhanced by including a spatially correlated residual. Can J For Res 31:1887-1893

Dutkowski GW, Costa e Silva J, Gilmour AR, Lopez GA (2002) Spatial analysis methods for forest genetic trials. Can J For Res 32:2201-2214
Dutkowski GW, Costa e Silva J, Gilmour AR, Wellendorf H, Aguiar AM (2006) Spatial analysis enhances modelling of a wide variety of traits in forest genetic trials. Can $\mathrm{J}$ For Res 36:1851-1870

Ericsson T (1997) Enhanced heritabilities and best linear unbiased predictors through appropriate blocking of progeny trials. Can J For Res 27:2097-2101

Fang L, Shi J (1998) Studies on comprehensive selection for superior family and clone of Chinese fir. J Nanjing For Univ 22(1):17-21 (Chinese with English abstract)

Gilmour AR, Cullis BR (1995) Average information reml: an efficient algorithm for variance parameter estimation in linear mixed models. Biometrics 51:1440-1450

Gilmour AR, Gogel BJ, Cullis BR, Thompson R (2009) ASReml user guide release 3.0. VSN International Ltd, Hemel Hempstead

Gleeson AC, Cullis BR (1987) Residual maximum likelihood (REML) estimation of a neighbour model for field experiments. Biometrics 43:277-288

Kusnandar D, Galwey N (2000) A proposed method for estimation of genetic parameters on forest trees without raising progeny: critical evaluation and refinement. Silvae Genet 49:15-21

Libby WJ, Cockerham CC (1980) Random non-contiguous plots in interlocking field layouts. Silvae Genet 29:183-190

Loo-Dinkins JA (1992) Field test design. In: Fins L, Friedman S, Brotschol J (eds) Handbook of quantitative forest genetics. Kluwer, Dordrecht, pp 96-139

Magnussen S (1990) Application and comparison of spatial models in analyzing tree-genetics field trials. Can J For Res 20:536-546

Magnussen S (1993) Bias in genetic variance estimates due to spatial autocorrelation. Theor Appl Genet 86:349-355

Martin RJ (1990) The use of time-series models and methods in the analysis of agricultural field trials. Commun Stat Theory Methods 19:55-81

Papadakis JS (1937) Méthode statistique pour des expériences sur champ. Bull Inst Amel Plantes Solonique 23:12-28

Patterson HD, Thompson R (1971) Recovery of inter-block information when block sizes are unequal. Biometrika 58:545-554

Shi J (1994) The Present situation of Chinese fir genetic improvement in Fujian Province and the technical countermeasures of developing it. J Fujian For Sci Technol 21(3):28-31 (Chinese with English abstract)

Smith A, Cullis B, Thompson R (2005) The analysis of crop cultivar breeding and evaluation trials: an overview of current mixed model approaches. J Agric Sci 143:449-462

White TL, Adams WT, Neale DB (2007) Forest genetics. CABI Publishing, Wallingford

Wilkinson GN, Eckert SR, Hancock TW, Mayo O (1983) Nearest neighbor (NN) analysis of field experiments. J R Stat Soc B 45:151-211

Williams ER, Matheson AC, Harwood CE (2002) Experimental design and analysis for tree improvement, 2nd edn. CSIRO, Collingwood

Ye TZ, Jayawickrama KJS (2008) Efficiency of using spatial analysis in first-generation coastal douglas-fir progeny tests in the US pacific northwest. Tree Genet Genomes 4:677-692

Yu R, Shi J, Liang Y, Chen X, Tan J, He Z (2001) The analysis of open pollination progeny tests of Cunninghamia lanceolata second-generation seed orchards. J Fujian For Sci Technol 28:16-18 (Chinese with English abstract) 